\title{
Nucleolar stress: is there a reverse version?
}

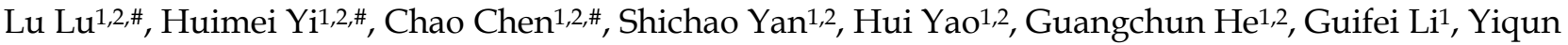 \\ Jiang1, Tuo Deng 3,4, Xiyun Deng $1,2, \bowtie$ \\ 1. Key Laboratory of Translational Cancer Stem Cell Research, Hunan Normal University, Changsha, Hunan 410013, China; \\ 2. Departments of Pathology and Pathophysiology, Hunan Normal University School of Medicine, Changsha, Hunan 410013, China; \\ 3. Diabetes Research Center and Center for Bioenergetics, Houston Methodist Research Institute, Houston, Texas 77030, USA; \\ 4. Department of Medicine, Weill Cornell Medical College at Cornell University, New York, New York 10021, USA. \\ \# These authors contributed equally to this work. \\ $\triangle$ Corresponding author: Xiyun Deng, Ph.D., Key Laboratory of Translational Cancer Stem Cell Research, Hunan Normal University, 371 Tongzipo Road, \\ Changsha, Hunan 410013. Telephone: + 867318891 2416; Fax: + 867318887 2260; E-mail: dengxiyunmed@hunnu.edu.cn \\ (c) Ivyspring International Publisher. This is an open access article distributed under the terms of the Creative Commons Attribution (CC BY-NC) license \\ (https://creativecommons.org/licenses/by-nc/4.0/). See http://ivyspring.com/terms for full terms and conditions.
}

Received: 2018.06.04; Accepted: 2018.08.04; Published: 2018.09.08

\begin{abstract}
The nucleolus is a dynamic structure that has roles in various physiological and pathophysiological processes. Perturbations on many aspects of the nucleolar functions are thought to cause "nucleolar stress", which occurs in response to a variety of chemotherapeutic drugs. The main characteristic changes of nucleolar stress include: 1) reduction of the size and volume of the nucleolus; 2) inhibition of RNA Pol I-mediated rRNA synthesis; and 3) nucleoplasmic translocation of nucleolar stress-related proteins. In studying the apoptosis-inducing effect of the natural compound lovastatin (LV) on breast cancer stem cells, we unexpectedly uncovered a novel form of nucleolar stress, which we call "reverse nucleolar stress". In our system, the canonical nucleolus stress inducer doxorubicin caused nucleoplasmic translocation of the nucleolar protein NPM and complete abolishment of Nolcl, an NPM-interacting protein and an activator of rRNA transcription. In contrast, the reverse nucleolar stress induced by LV is manifested as a more localized perinucleolar distribution of NPM and an increase in the protein level of Nolcl. Furthermore, translocation of the ribosomal protein RPL3 from the cytoplasm to the nucleolus and increased AgNOR staining were observed. These changes characterize a novel pattern of nucleolar stress doubtlessly distinguishable from the canonical one. The functional consequences of reverse nucleolar stress are not clear at present but may presumably be related to cell death or even normalization of the stressed cell. The discovery of reverse nucleolar stress opens up a new area of research in molecular and cellular biology and might have important implications in cancer therapy.
\end{abstract}

Key words: Nucleolus, nucleolar stress, ribosome biogenesis

\section{Introduction}

The nucleolus is the most prominent membraneless structure in the nucleus of eukaryotic cells. The nucleolus is formed around nucleolar organizer region (NOR), which is crucial for the dynamic formation of the nucleolus during the cell cycle [1]. The ultrastructure of the nucleolus was revealed by electron microscopy to be a tripartite structure, which is composed of the fibrillar center (FC), the dense fibrillar component (DFC), and the granular component (GC) [2, 3]. During ribosomal RNA (rRNA) transcription, the ribosomal DNA
(rDNA) is initially transcribed into the 47S rRNA precursor by RNA polymerase I (RNA Pol I). This 47S rRNA transcript is subsequently cleaved and processed into $18 \mathrm{~S}, 5.8 \mathrm{~S}$, and $28 \mathrm{~S}$ rRNAs, which, together with $5 \mathrm{~S}$ rRNA that is transcribed from RNA Pol III, are assembled with various ribosomal proteins before interaction with the export machinery and transport to the cytoplasm to form the large and small subunits of the ribosome $[4,5]$. The nucleolus has long been known to be the site of ribosome biogenesis or the factory of the ribosome. In the last three decades, 
the nucleolus has been shown to be the site of many new functions including signal recognition particle assembly, small RNA modification, telomerase maturation, cell cycle and aging control, and cell stress sensing [6-8]. More intriguingly, targeting the nucleolus has recently shown great promise and holds significant clinical implications in cancer therapy [9]. These functions of the nucleolus, especially the non-traditional ones, make the study of nucleolus a very attractive and active area of investigations in molecular and cellular biology and cancer therapeutics.

\section{Nucleolar stress: the canonical pattern}

The nucleolus carries a heavy metabolic burden that makes the nucleolar and ribosomal functions, which are required for protein synthesis and cell growth, extremely sensitive to external stimuli. Perturbations of ribosome biogenesis, accompanied by structural and functional disorders of the nucleolus, cause nucleolar stress (also called ribosomal stress), which leads to disruptions of cell homeostasis and eventual cell death [10, 11]. Nucleolar stress has a major impact on fundamental cellular functions for at least the following two reasons. First, about $80 \%$ of a cell's energy is dedicated to ribosome biogenesis in an actively cycling eukaryotic cell [12]. Second, almost all metabolic and signaling pathways in a cell are related with the nucleolus [13]. Nucleolar stress often leads to down-regulation of the nucleolar functions and a series of biological behavior changes of the cell, including disorders of protein translation, cell cycle arrest, and cell death $[14,15]$.

The main characteristic changes of nucleolar stress include: 1) reduction in the number of nucleoli and/or disintegration of the nucleolar structure; 2) inhibition of RNA Pol I-mediated rRNA synthesis, processing, and subsequent disturbance of ribosomal assembly and protein translation; and 3) translocation of nucleolar stress-related proteins including nucleolar proteins (e.g., NPM, Nolc1, fibrillarin) and free ribosomal proteins (e.g., RPL3) from the nucleolus to the nucleoplasm and/or the cytoplasm [16-18]. Among these, translocation of NPM from the nucleolus to the nucleoplasm is considered the most typical hallmark of canonical nucleolar stress [17, 19, 20]. These changes are usually used as parameters for the assessment of nucleolar stress in various experimental systems.

\section{Reverse nucleolar stress: an unexpected discovery}

Lovastatin (LV) is a natural compound derived from extracts of Monascus-fermented foods and an
FDA-approved drug used to treat hyperlipidemia [21]. Recently, the anti-cancer effects of LV have been called into attention by our group [22-24] and others $[25,26]$. In further studying the cellular and molecular actions of LV, we unexpectedly discovered a novel form of nucleolar stress, which distinguishes itself from canonical nucleolar stress in several ways as described below. First, we observed a reposition of NPM from a more scattered distribution in the nucleolus in untreated cells to a more localized perinucleolar distribution within the nucleolus in LV-treated cells. In contrast, doxorubicin (DXR), a chemotherapeutic drug commonly used to treat a variety of solid and hematopoietic malignancies [27] and a classical nucleolar stress inducer [28], caused NPM translocation from the nucleolus to the nucleoplasm, confirming the occurrence of canonical nucleolar stress (Figure 1A) [17, 19, 29]. Next, we examined the effect of LV on nucleolar and coiled-body phosphoprotein 1 (Nolc1/Nopp140), an NPM-interacting protein [30] and an activator of rRNA transcription [31]. It is known that DXR induces nucleolar stress through targeting Nolc1 [32]. DXR expectedly diminished Nolc1 in the nucleolus, consistent with its well-established role in inducing nucleolar stress. We investigated whether LV had a similar effect on Nolc1. To our surprise again, we did not observe a decrease but rather an increase in Nolc1 in the nucleolus after LV treatment (Figure 1B).

NPM functions as a molecular chaperone facilitating the transport of ribosomal proteins such as RPL3 from the nucleolus to the nucleoplasm and/or cytoplasm [33] and Nolc1 is involved in the activation of RNA Pol I transcription [31]. Therefore, we examined whether LV would affect the intracellular localization of RPL3 and the rRNA transcriptional activity by laser scanning confocal microscopy and argyrophilic nucleolar organizer region (AgNOR) staining. As expected, we found that LV induced a backward flow from the cytoplasm to the nucleus especially to the nucleolus (Figure 1C). Silver staining of AgNOR is closely related to and can be used as a surrogate for rRNA transcriptional activity [34]. Similarly, we found an increase, rather than a decrease, in AgNOR staining in LV-treated cells (Figure 1D).

To address the issue whether other statins have similar effects in inducing reverse nucleolar stress, we compared between LV and Atorvastatin (AV) and Simvastatin (SV), two other commonly used lipophilic statins. We found that all three lipophilic statins similarly induced perinucleolar distribution of NPM and increased the protein level of nucleolar Nolc1 in MDA-MB-231 cancer stem cells (Figure S1). In addition, we found all three statins have similar 
A

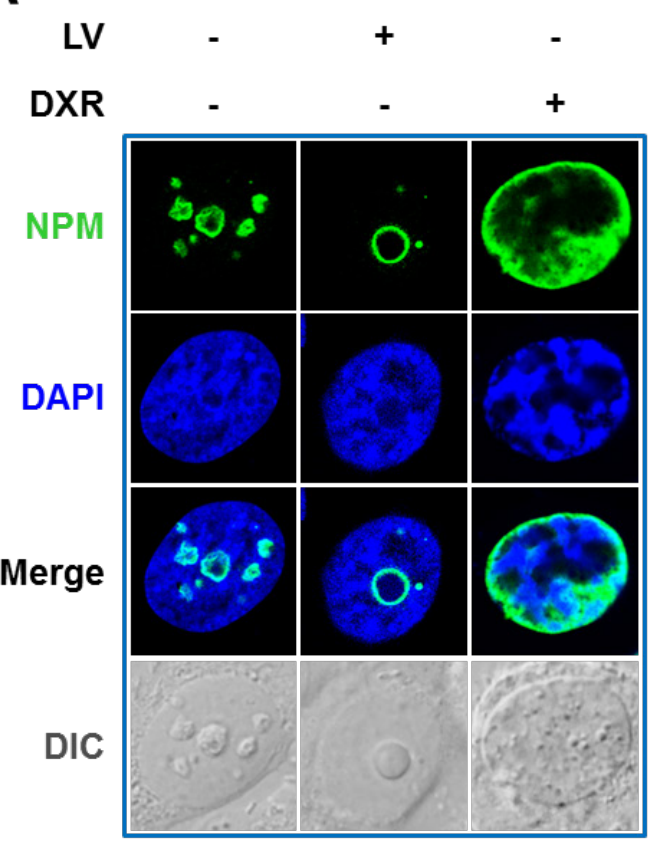

B

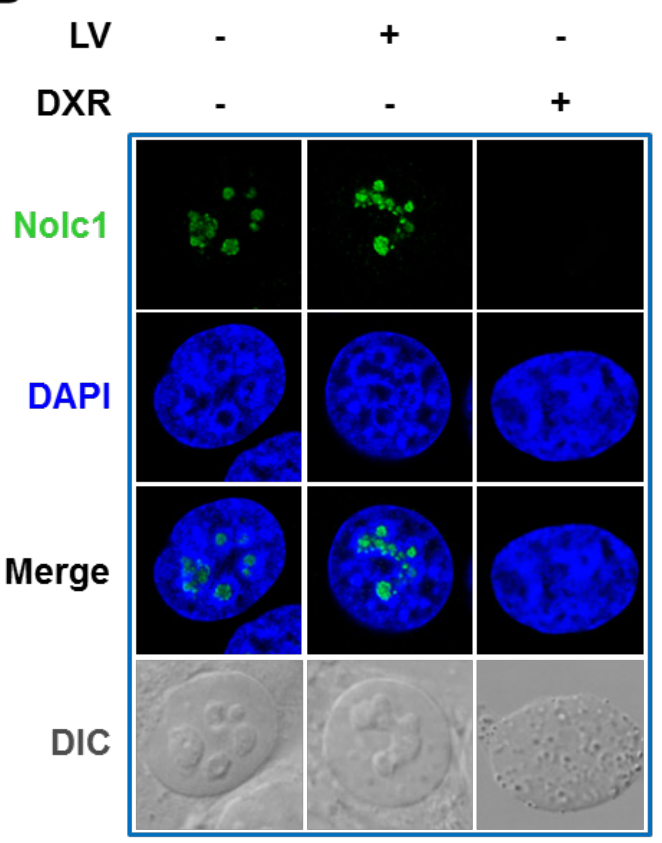

C

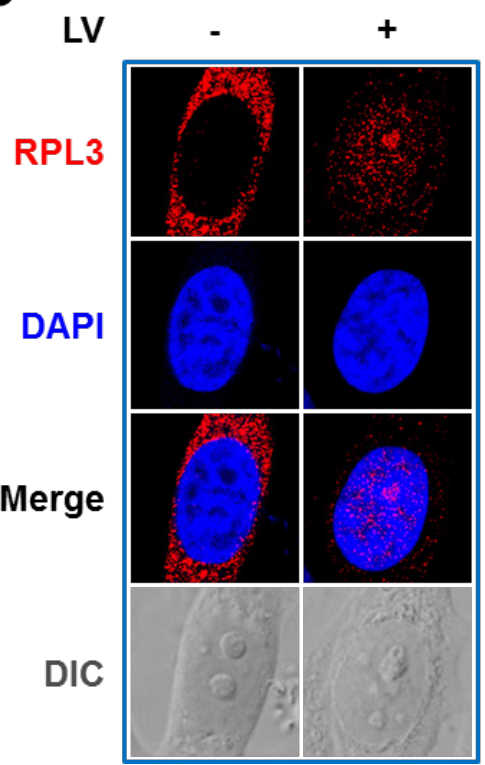

D

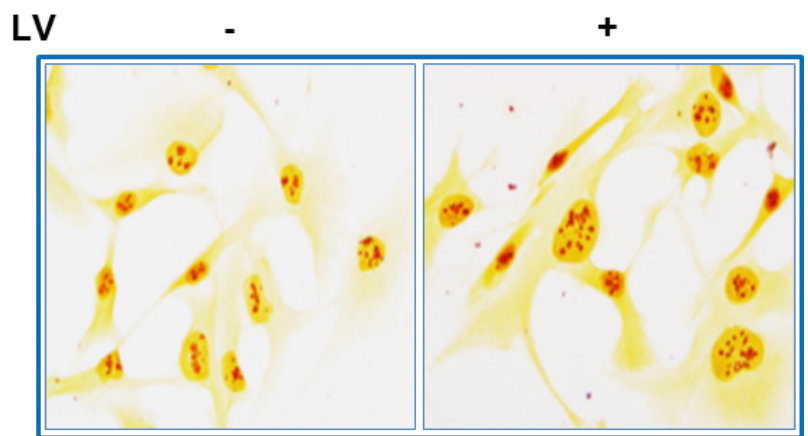

Figure 1. LV triggers reverse nucleolar stress. Immunofluorescence-laser confocal scanning microscopic observation of the intracellular localization of NPM (A), Nolcl (B), and RPL3 (C). MDA-MB-231 cancer stem cells were treated with LV or DXR for $48 \mathrm{~h}$, fixed, stained using an anti-NPM or anti-Nolc1 antibody, and then subjected to laser confocal microscopy. A. LV induced a reposition of NPM from a more scattered distribution in the nucleolus in untreated cells to a more localized perinucleolar distribution within the nucleolus of treated cells. DXR contrarily induced nucleoplasmic translocation of NPM, characteristic of canonical nucleolar stress. B. LV increased the protein level of nucleolar Nolcl; whereas DXR completely abolished it. C. LV induced translocation of the ribosomal protein RPL3 from cytoplasm to the nucleus and the nucleolus. D. Silver staining of argyrophilic nucleolar organizer region (AgNOR). MDA-MB-231 cancer stem cells were treated with LV or vehicle for $12 \mathrm{~h}$, fixed and stained with silver nitrate, then subjected to microscopic examination. LV could increase the NOR staining intensity in MDA-MB-231 cancer stem cells, suggestive of increased RNA Pol I transcriptional activity. Differential interference contrast (DIC) microscopy was used to reveal the morphology of the nucleolus. LV, lovastatin; DXR, doxorubicin; AU, arbitrary unit.

inhibitory effect on the viability of MDA-MB-231 cancer stem cells (data not shown). These data suggest that the reverse nucleolar stress we observed is not specific for LV and may be a shared phenomenon of all lipophilic statins.

Taken together, our observations point to a previously unrecognized or overlooked stress response of the nucleolus, which is termed reverse nucleolar stress. This novel type of nucleolar stress is different from the canonical one in several ways. First, NPM is not translocated from the nucleolus to the nucleoplasm but rather distributed to the perinucleolar zone and possibly functions to transport ribosomal proteins such as RPL3 back to the 


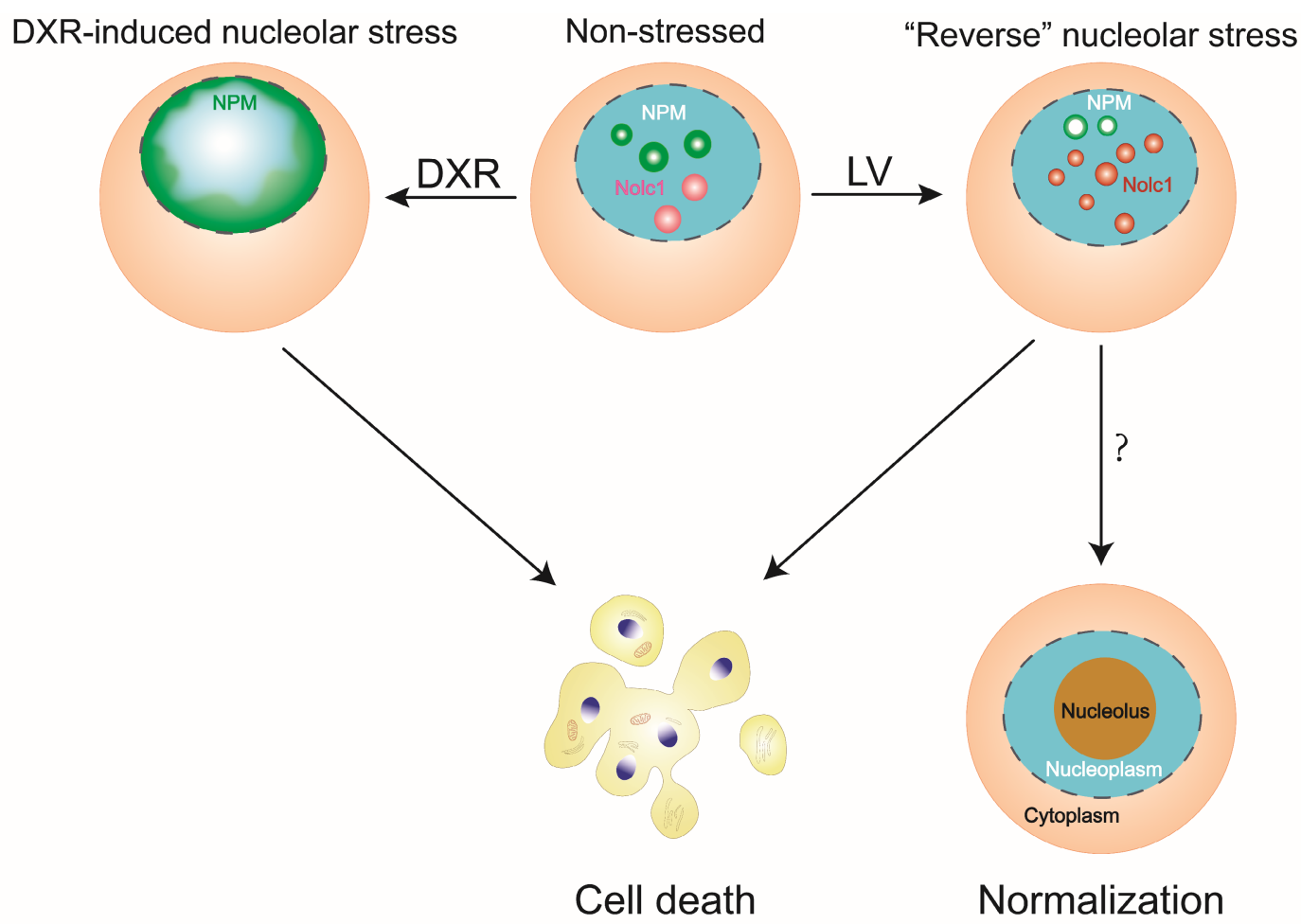

Figure 2. A diagram depicting reverse nucleolar stress in comparison with canonical nucleolar stress. In reverse nucleolar stress, LV induces a reposition of NPM from a scattered nucleolar distribution to a perinucleolar distribution and an increase in nucleolar Nolcl. In canonical nucleolar stress, DXR causes nucleoplasmic translocation of NPM and abolishment of nucleolar Nolcl. Both types of nucleolar stress may lead to eventual cell death. In reverse nucleolar stress, the possibility exists that the cell becomes less malignant, leading to normalization of the cellular structure and function.

nucleolus. Second, Nolc1 is not diminished but increased in the nucleolus, which possibly exerts its rRNA transcription-promoting effect (Figure 2). The changes of reverse nucleolar stress are definitely distinguishable from its canonical counterpart in terms of NPM localization and Nolc1 protein level.

In its canonical setting, nucleolar stress results in the inhibition of nucleolar functions, leading to disruption of the cellular homeostasis and eventual cell death. The reverse nucleolar stress we describe here, obviously, triggers a different set of stress responses of the nucleolus, some seemingly stimulatory rather than inhibitory. However, we don't know whether these reverse changes will stimulate or possibly stabilize nucleolar activity and whether they are related with enhanced ribosomal activity. Nevertheless, aberrant stimulation or inhibition of the nucleolar function may both similarly cause disturbance of the cellular homeostasis, leading to cellular disaster such as cell death.

\section{Perspectives}

Nucleolar stress has been the focus of intense investigations for many years. Several possibilities exist to explain the similarities and differences between the canonical type and the reverse type of nucleolar stress. It is likely that both canonical and reverse nucleolar stress responses share a similar final outcome, i.e., increased cell death. However, even though the cell death rate increases as a whole, the individual cells that undergo reverse nucleolar stress may show different fates. These cells may either die or survive the harsh conditions generated by the stress inducer. In the latter case, reverse nucleolar stress might provide an opportunity for the cell to become less malignant, leading to phenotypic reversal toward normalization. These different possibilities need to be studied empirically and are the focus of our ongoing investigations.

Furthermore, several important issues concerning how, why, and at what point these two types of nucleolar stress differ from each other are not clear and are worthy of further investigations. First, as mentioned above, does reverse nucleolar stress lead to promotion rather than inhibition of nucleolar functions? Or it is simply an unusual manifestation of similar dysfunctions of the nucleolus as observed in canonical nucleolar stress? Second, is this reverse nucleolar stress a stressor-dependent effect? If yes, hopefully a stress-inducing agent triggers either canonical or reverse nucleolar stress depending on its physicochemical properties. Third, what is the molecular determinant that discriminates reverse nucleolar stress from the canonical one and what is the point of convergence that serves to drive similar downstream responses between the two types of 
nucleolar stress? Despite all these unsolved issues, our preliminary findings open up a new area of molecular and cellular biology research and possibly mark a new era of investigating the non-traditional regulation of nucleolar functions.

\section{Abbreviations}

AgNOR: Argyrophilic nucleolar organizer region; CSCs: Cancer stem cells; DXR: Doxorubicin; LV: Lovastatin; Nolc1: Nucleolar and coiled-body phosphoprotein 1; NOR: Nucleolar organizer region; NPM: Nucleophosmin; rDNA: Ribosomal DNA; RNA Pol I: RNA polymerase I; RPL: Ribosomal protein large subunit; RPS: Ribosomal protein small subunit; rRNA: Ribosomal RNA; TNBC: Triple-negative breast cancer.

\section{Supplementary Material}

Supplementary figure.

http://www.jcancer.org/v09p3723s1.pdf

\section{Acknowledgements}

This work was supported by the National Natural Science Foundation of China (81472496) and the Key Project of Department of Education of Hunan Province (14A089).

\section{Competing Interests}

The authors have declared that no competing interest exists.

\section{References}

[1] Nemeth A, Grummt I. Dynamic regulation of nucleolar architecture. Curr Opin Cell Biol. 2018;52:105-11.

[2] Lo SJ, Lee CC, Lai HJ. The nucleolus: reviewing oldies to have new understandings. Cell Res. 2006;16:530-8.

[3] Hiscox JA. RNA viruses: hijacking the dynamic nucleolus. Nat Rev Microbiol. 2007;5:119-27.

[4] Boisvert FM, van Koningsbruggen S, Navascues J, Lamond AI. The multifunctional nucleolus. Nat Rev Mol Cell Biol. 2007;8:574-85.

[5] Goodfellow SJ, Zomerdijk JC. Basic mechanisms in RNA polymerase I transcription of the ribosomal RNA genes. Subcell Biochem. 2013;61:211-36.

[6] Ma TH, Lee LW, Lee CC, Yi YH, Chan SP, Tan BC, et al. Genetic control of nucleolar size: An evolutionary perspective. Nucleus. 2016;7:112-20.

[7] Olson MO. Sensing cellular stress: another new function for the nucleolus? Sci STKE. 2004;2004:pe10.

[8] Quin JE, Devlin JR, Cameron D, Hannan KM, Pearson RB, Hannan RD. Targeting the nucleolus for cancer intervention. Biochim Biophys Acta. 2014;1842:802-16.

[9] Hein N, Hannan KM, George AJ, Sanij E, Hannan RD. The nucleolus: an emerging target for cancer therapy. Trends Mol Med. 2013;19:643-54.

[10] Suzuki A, Kogo R, Kawahara K, Sasaki M, Nishio M, Maehama T, et al. A new PICTure of nucleolar stress. Cancer Sci. 2012;103:632-7.

[11] Wang W, Nag S, Zhang X, Wang MH, Wang H, Zhou J, et al. Ribosomal proteins and human diseases: pathogenesis, molecular mechanisms, and therapeutic implications. Med Res Rev. 2015;35:225-85.

[12] Moss T, Stefanovsky VY. At the center of eukaryotic life. Cell. 2002;109:545-8.

[13] Vlatkovic N, Boyd MT, Rubbi CP. Nucleolar control of p53: a cellular Achilles' heel and a target for cancer therapy. Cell Mol Life Sci. 2014;71:771-91.

[14] Orsolic I, Jurada D, Pullen N, Oren M, Eliopoulos AG, Volarevic S. The relationship between the nucleolus and cancer: Current evidence and emerging paradigms. Semin Cancer Biol. 2016;37-38:36-50.

[15] Golstein P. Conserved nucleolar stress at the onset of cell death. FEBS J. 2017:284:3791-800.

[16] Lewinska A, Bednarz D, Adamczyk-Grochala J, Wnuk M. Phytochemical-induced nucleolar stress results in the inhibition of breast cancer cell proliferation. Redox Biol. 2017;12:469-82.
[17] Fu X, Xu L, Qi L, Tian H, Yi D, Yu Y, et al. BMH-21 inhibits viability and induces apoptosis by p53-dependent nucleolar stress responses in SKOV3 ovarian cancer cells. Oncol Rep. 2017;38:859-65.

[18] Russo A, Russo G. Ribosomal Proteins Control or Bypass p53 during Nucleolar Stress. Int J Mol Sci. 2017;18.

[19] Yang K, Wang M, Zhao Y, Sun X, Yang Y, Li X, et al. A redox mechanism underlying nucleolar stress sensing by nucleophosmin. Nat Commun. 2016;7:13599.

[20] Lindstrom MS, Zhang Y. B23 and ARF: friends or foes? Cell Biochem Biophys. 2006;46:79-90.

[21] Yang T, Liu J, Luo F, Lin Q, Rosol TJ, Deng X. Anticancer properties of Monascus metabolites. Anticancer Drugs. 2014;25:735-44.

[22] Song L, Tao X, Lin L, Chen C, Yao H, He G, et al. Cerasomal Lovastatin Nanohybrids for Efficient Inhibition of Triple-Negative Breast Cancer Stem Cells To Improve Therapeutic Efficacy. ACS Appl Mater Interfaces. 2018;10:7022-30.

[23] Peng Y, He G, Tang D, Xiong L, Wen Y, Miao X, et al. Lovastatin Inhibits Cancer Stem Cells and Sensitizes to Chemo- and Photodynamic Therapy in Nasopharyngeal Carcinoma. J Cancer. 2017;8:1655-64.

[24] Yao H, He G, Yan S, Chen C, Song L, Rosol TJ, et al. Triple-negative breast cancer: is there a treatment on the horizon? Oncotarget. 2017;8:1913-24.

[25] Campbell MJ, Esserman LJ, Zhou Y, Shoemaker M, Lobo M, Borman E, et al. Breast cancer growth prevention by statins. Cancer Res. 2006;66:8707-14.

[26] Rao S, Porter DC, Chen X, Herliczek T, Lowe M, Keyomarsi K. Lovastatin-mediated G1 arrest is through inhibition of the proteasome, independent of hydroxymethyl glutaryl-CoA reductase. Proc Natl Acad Sci U S A. 1999;96:7797-802.

[27] Gabizon AA, Patil Y, La-Beck NM. New insights and evolving role of pegylated liposomal doxorubicin in cancer therapy. Drug Resist Updat. 2016;29:90-106.

[28] Woods SJ, Hannan KM, Pearson RB, Hannan RD. The nucleolus as a fundamental regulator of the p53 response and a new target for cancer therapy. Biochim Biophys Acta. 2015;1849:821-9.

[29] Kurki S, Peltonen K, Latonen L, Kiviharju TM, Ojala PM, Meek D, et al. Nucleolar protein NPM interacts with HDM2 and protects tumor suppressor protein p53 from HDM2-mediated degradation. Cancer Cell. 2004;5:465-75.

[30] Li Z, Hann SR. The Myc-nucleophosmin-ARF network: a complex web unveiled. Cell Cycle. 2009;8:2703-7.

[31] Yuan F, Zhang Y, Ma L, Cheng Q, Li G, Tong T. Enhanced NOLC1 promotes cell senescence and represses hepatocellular carcinoma cell proliferation by disturbing the organization of nucleolus. Aging Cell. 2017;16:726-37.

[32] Jin Y, Yu J, Yu YG. Identification of hNopp140 as a binding partner for doxorubicin with a phage display cloning method. Chem Biol. 2002;9:157-62.

[33] Szebeni A, Hingorani K, Negi S, Olson MO. Role of protein kinase CK2 phosphorylation in the molecular chaperone activity of nucleolar protein b23. J Biol Chem. 2003;278:9107-15.

[34] McStay B. Nucleolar organizer regions: genomic 'dark matter' requiring illumination. Genes Dev. 2016;30:1598-610. 